\title{
Entretien avec Krzysztof Warlikowski
}

Contes africains d'après Shakespeare, Théâtre de Chaillot (mars 2012)

Krzysztof Warlikowski, Catherine Treilhou-Balaudé, Lucie Vérot et Christophe Hausermann

\section{(2) OpenEdition \\ 12 Journals}

\section{Édition électronique}

URL : http://journals.openedition.org/shakespeare/1965

DOI : 10.4000/shakespeare. 1965

ISSN : 2271-6424

Éditeur

Société Française Shakespeare

\section{Édition imprimée}

Date de publication : 1 avril 2013

Pagination : 239-244

ISBN : 2-9521475-9-0

\section{Référence électronique}

Krzysztof Warlikowski, Catherine Treilhou-Balaudé, Lucie Vérot et Christophe Hausermann, «Entretien avec Krzysztof Warlikowski », Actes des congrès de la Société française Shakespeare [En ligne],

30 | 2013, mis en ligne le 03 avril 2013, consulté le 30 avril 2019. URL : http://journals.openedition.org/ shakespeare/1965; DOI : 10.4000/shakespeare.1965 


\section{Shakespeare et la mémoire}

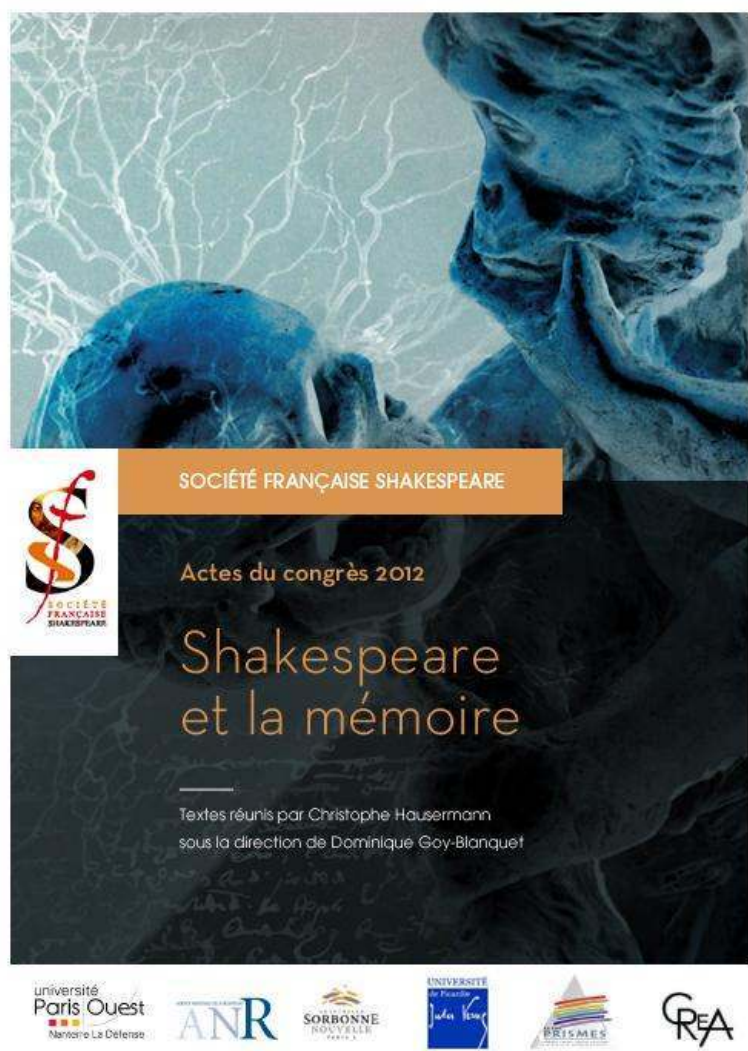

actes du Congrès

organisé par la

SOCIÉTÉ FRANÇAISE SHAKESPEARE

les 22, 23 et 24 mars 2012

textes réunis par

Christophe HAUSERMANN

sous la direction de

Dominique GoY-BLANQUET 


\section{COUVERTURE}

d'après l'affiche de Claire Colombet

conception graphique et logo

Pierre Kapitaniak

mise en page et corrections

Christophe Hausermann

(C) 2012 Société Française Shakespeare

Institut du Monde Anglophone

Université de Paris III - Sorbonne Nouvelle

5 rue de l'École de Médecine 75006 Paris

www.societefrancaiseshakespeare.org

Tous droits de traduction, de reproduction et d'adaptation réservés pour tous les pays 


\section{Entretien AVEC Krzysztof WARLIKOWSKI ${ }^{1}$}

Catherine Treilhou-Balaudé: Krzysztof Warlikowski, vous venez de mettre en scène avec l'équipe du Nowy Teatr $^{2}$ la pièce Contes africains ${ }^{3}$ d'après les pièces de Shakespeare (Le Roi Lear, Othello et Le Marchand de Venise), les romans de J. M. Coetzee (L'Été de la vie et Au cour de ce pays), les essais d'Eldridge Cleaver (Soul of Ice) et des textes originaux de Wajdi Mouawad. Shakespeare semble être le fil conducteur de vos productions théâtrales. Vous avez mis en scène Le Marchand de Venise, Le Conte d'hiver, Macbeth, en 2001 Hamlet à Avignon, La Mégère apprivoisée, Péricles, La Nuit des rois, La Tempête et Le Songe d'une nuit d'été. Dans (A)pollonia, vous aviez déjà confronté Euripide, Eschyle, Hanna Krall, Jonathan Littell et Coetzee. Dans Contes africains, vous inventez un théâtre dans lequel Shakespeare est présent, mais où les problématiques des pièces originales sont éloignées dans le temps. Pourquoi revenir maintenant à Shakespeare alors que vous aviez annoncé que vous ne monteriez plus aucune de ses pièces?

Krzysztof Warlikowski : Contes africains ne ressemble pas à ce que j'ai fait auparavant. Shakespeare est mon maître. C'est avec lui que j'ai appris à faire du théâtre, ce fut un lent trajet. Ce que Shakespeare propose me bouleverse toujours, mais à présent je ne me retrouve dans aucune de ses pièces prise individuellement. Je monte des pièces à un rythme long et régulier (un spectacle tous les deux ans en moyenne). Le défi est différent pour moi. Avec Contes africains, je voulais me placer du point de vue de la minorité, dont je me revendique. On se considère toujours en tant que minorité. À travers les minorités on peut mieux comprendre le monde. J'ai tout d'abord choisi les trois pièces les plus politiquement engagées de Shakespeare: Othello, Le Marchand de

\footnotetext{
1 Réalisé le vendredi 23 mars 2012 par Catherine Treilhou-Balaudé et Lucie Vérot à l'occasion du spectacle Contes africains au Théâtre de Chaillot dans le cadre du congrès de la Société Française Shakespeare "Shakespeare et la mémoire », INHA, 6, rue des Petits Champs, 75002 Paris.

2 http://www.nowyteatr.org/\#/pl.

3 Communiqué de presse de la pièce Contes africains :

http://www.societefrancaiseshakespeare.org/docannexe.php?id=1676
} 
Venise et La Mégère apprivoisée. Le Roi Lear a finalement remplacé cette dernière, car j'ai trouvé drôle de penser que le vieillard faisait partie d'une minorité.

Lucie Vérot: Contes africains dépasse les minorités politiques. La pièce n'exprime-t-elle pas la marginalité des désirs ? Shylock apparaît comme un fantasme de l'anthropologie.

$K W$ : Contes africains est une trilogie. Je voulais donner des versions excentriques de chacune des pièces de Shakespeare, ce qui me donnait plus de liberté. Contes africains s'ouvre sur la scène du partage de l'héritage dans Le Roi Lear. Cela commence par une question: " comment vous m'aimez? ». C'est une question abusive et étrange. J'étais intéressé par ce père qui ne peut pas se séparer de sa fille. Le monologue de Cordelia travaillé avec Wajdi Mouawad laisse d'ailleurs penser qu'il y a eu inceste. Cette question fait écho aux souvenirs d'enfance. Un jour on m'a demandé : « Lequel tu préfères ? Ton père ou ta mère? "J'avais honte, je ne savais pas comment répondre. Finalement j'ai choisi ma mère.

Ensuite il y a Shylock. En Pologne, Le Marchand de Venise n'a jamais été joué après la guerre alors qu'il y avait eu de nombreuses représentations à Varsovie avant la guerre. J'avais essayé de le mettre en scène quand j'étais jeune. Par la suite, j'ai eu plus de recul, plus « d'instruments » après avoir monté les pièces de Sarah Kane, de Tony Kushner et de Bernard-Marie Koltès pour revenir au Marchand. Dans Contes africains, Shylock est présenté comme un boucher. La mémoire $\mathrm{du} \mathrm{XX}^{\mathrm{e}}$ siècle est trop forte. Donc, il fallait faire attention dans la mise en scène pour ne pas paraître antisémite. Le boucher ensanglanté allégeait le caractère choquant de la livre de chair. Il fallait se confronter à ce « hors-social ». Shylock aurait dû se rendre compte de l'absurdité de sa demande, même si la loi de l'époque l'y autorisait. Vengeance, humiliation, guerre au monde entier ? Il y a tout cela dans Contes africains, mais aussi un deal pas propre, un deal pervers. Je voulais voir comment le personnage vivait avec ça.

$C T B$ : D’où l'épaisseur que vous donnez au voyeurisme dans la pièce. Shylock semble jouir de son incarcération, on lui apporte à manger 
comme pour le faire grossir, comme s'il était une bête que l'on engraisse avant de l'abattre.

$K W$ : Je vous ai donné quelques «traces" pour que vous fassiez le voyage. À vous de découvrir le reste. Pour en finir avec cette introduction, j'aimerais parler d'Othello. Chez Shakespeare, Othello et Desdémone n'ont pas l'air de se rendre compte de la singularité du mariage entre une blanche et un noir. On ne fait pas attention. Othello est un black qui fait carrière (comme dans l'armée américaine), mais à un moment il touche une femme blanche, ce qui provoque un scandale. Ça, c'est l'aventure qui commence. Le drame c'est qu'il se rend compte de ce qu'il est. L'humiliation à Venise exclut ce rêve qu'il nous est donné de voir : le couple des jeunes mariés partant en voyage de noce. On se retrouve dans la chambre après la guerre. Othello lutte pour cette société. Je trouvais dommage d'abandonner cette trace pour n'aborder que la jalousie, qui n'est que la deuxième trame de la pièce, à partir de l'acte II.

$C T B$ : Le mariage catholique est violent par nature.

$K W$ : Il y a des détails qui crient dans le silence. Dans Contes africains, à partir du mariage d'Othello et Desdémone, les deux amoureux finissent la scène dans une salle de bains. Ils sont alors confrontés à leur vie future. Je connais des filles blanches qui ne veulent être en couple qu'avec des blacks, car elles ne veulent pas se faire dominer par un blanc. Avec un black, elles ont l'impression d'avoir le dessus.

CTB : Pour le titre de la pièce, vous avez choisi le mot « conte » ? La livre de chair, les coffrets et les autres éléments des pièces de Shakespeare sont juxtaposés à un contexte africain. Dans quelle mesure ? Quel rôle joue l'Afrique dans Contes africains?

$K W$ : J'ai effectivement juxtaposé le conte et l'Afrique. À vous de voir ce que vous pouvez ajouter à cette juxtaposition.

$L V$ : La mémoire et l'oubli sont des thèmes qui ont hanté votre travail, notamment la mémoire collective et le traumatisme individuel. 
$K W$ : La mémoire c'est énorme, inabordable quand on parle de Shakespeare.

$C T B$ : Vous faites plus de l'anti-théâtre que du théâtre. Dans vos mises en scène, le cinéma est présent. Dans la pièce, Desdémone regarde un film de Kurosawa. On retrouve également dans la pièce la présence très troublante de l'animal : le porc, mais surtout le chien, qui apparaît dans une vidéo surprenante. L'animal met en danger l'humain et questionne notre humanité. Il introduit également une part d'aléatoire.

$K W$ : La chair est très présente dans la pièce. Entre l'animal et l'humain, il y a un abîme. Mais, Desdémone parle à un chien parce qu'elle n'a plus personne à qui parler.

$L V:$ Pourtant elle a une dame de compagnie.

$K W$ : Elle ne lui parle pas vraiment. Elle la renvoie.

\section{QUESTIONS DU PUBLIC}

Question : À propos de Lear, peu de critiques ont souligné l'absence de la mère. Or, ce qui est dans les limbes du spectacle, ce qui est laissé en suspens, a souvent une importance capitale dans la pièce.

$K W$ : C'est vrai. Le manque de la mère est très fort dans Lear. Desdémone est quant à elle rejetée par son père.

Question : Contes africains se présente comme un enchaînement. À cet égard, la performance théâtrale de l'acteur principal (Adam Ferency) est remarquable. Mais, la pièce présente-t-elle un discours sur l'homme ? Tous ces personnages se retrouvent-ils dans un seul être?

$K W$ : Ma première idée fut de savoir comment, à un certain moment de ma vie, je suis passé par une crise, comment j'ai pressenti la fin, la mort, la peur, je voulais donner cette image de l'homme. Shakespeare et Coetzee ont deux points de vue différents sur l'homme. Il y a un discours féminin dans L'Été de ma vie, une interview des femmes de 
Coetzee qui témoignent après sa mort fictive. L'une d'elles raconte sa relation avec lui. Elle pensait «c'était le mec de ma vie, mais il a eu peur et il est parti dans la nuit ». Dans les pièces de Shakespeare, on nous montre que l'on est né pour être un garçon sans nous dire comment on fait pour devenir un homme: l'honneur, les responsabilités, le fait d'être un père, un mari. Le mec n'est pas au niveau. On se veut héros. Chaque homme naît avec une mémoire qui est très lourde de ce que l'on devrait être. Les femmes vont me dire plus de choses intéressantes sur l'homme (comme dans les romans de Coetzee).

Question: Vous présentez un spectacle total. Certains passages shakespeariens sont magiques, mais je regrette la violence masculine dans la pièce. Shakespeare est l'androgyne : la violence radicale est plus humaine que masculine. Les scènes de sexualisation apparaissent entre les extraits des pièces de Shakespeare et celles-ci semblent complètement dissociées. Ce n'est pas un spectacle pour femmes.

$K W$ : Shakespeare est androgyne. Mais pas Orlando. Shakespeare c'est la chair humaine.

Question: Desdémone dans Contes africains est plus un fantasme masculin que Desdémone dans Othello, notamment dans la scène où elle danse sous le regard des hommes. Vous présentez l'univers des hommes tel qu'ils se l'imaginent.

$K W$ : Cela me fait penser à un film de Fassbinder qui parle de la relation qu'entretient une femme allemande avec un jeune Turc ${ }^{4}$. Aujourd'hui, cette relation ne nous choque plus autant qu'à la sortie du film.

Question: Dans la scène de danse finale, on obéit à l'autorité féminine. $\mathrm{Au}$ début de cette scène, j'ai senti que les spectateurs se moquaient de l'actrice qui animait le cours de salsa 5 , notamment de son physique, mais elle a finalement réussi à retourner le public pour se faire respecter et le faire participer.

\footnotetext{
4 Tous les autres s'appellent Ali (Angst essen Seele auf, 1973).

5 Stanisława Celińska.
} 
$K W$ : Son corps lourd c'est aussi sa force. Sa présence fait du bien au spectateur. La salsa permet de rompre la solitude. Beaucoup de personnes vont prendre des cours pour faire des rencontres. C'est de la folie, les cours de salsa sont un terrain de drague, comme d'autres lieux très différents : les cours de langues, la rue, les chiottes.

Retranscrit par Christophe Hausermann 